\title{
Roadmap of 1300-nm InAs/GaAs quantum dot laser grown on silicon for silicon photonics
}

\author{
Zizhuo Liu ${ }^{1}$, Mengya Liao ${ }^{1}$, Constanze Hantschmann ${ }^{2}$, Yi Wang ${ }^{3}$, Taojie Zhou ${ }^{4}$, Mingchu Tang ${ }^{1}$, Jae-Seong \\ Park $^{1}$, Zhaoyu Zhang ${ }^{4}$, Siming Chen ${ }^{1}$, Alwyn Seeds ${ }^{1}$, Richard Penty ${ }^{2}$, Siyuan Yu ${ }^{3}$ and Huiyun Liu ${ }^{1}$ \\ 1. Department of Electronic and Electrical Engineering, University College London, London, WC1E 7JE, United Kingdom \\ 2. Centre for Photonic Systems, Department of Engineering, University of Cambridge, 9 JJ Thomson Avenue, Cambridge CB3 OFA, United \\ Kingdom \\ 3. State Key Laboratory of Optoelectronic Materials and Technologies, Sun Yat-sen University, Guangzhou 510275, China \\ 4. School of Science and Engineering, The Chinese University of Hong Kong, Shenzhen, Guangdong, 518172, China \\ Author e-mail address: zizhuo.liu.16@ucl.ac.uk
}

\begin{abstract}
Quantum dot (QD) lasers monolithically grown on silicon substrates have been considered as a promising approach for on-chip light sources in Si photonics. In this paper, the recent progress in Si-based InAs/GaAs QD lasers at 1300nm wavelength is demonstrated.

OCIS codes: (140.3460) Lasers, (230.2090) Electro-optical devices.
\end{abstract}

\section{Introduction}

Increasing data throughput in modern information processing requires unprecedented bandwidth and low power consumption beyond the capability of conventional copper interconnects. To meet these requirements, silicon photonics has been under intensive study in recent years [1, 2]. However, Si-based light-emitting sources have been regarded as "holy grail" for Si-based photonic integrated circuits and opto-electronic integrated circuits because of the in-direct bandgap property of bulk Si material, which has very low efficiency on photon emission. Even though researchers have developed Ge alloy laser and Si Raman laser, extremely high thresholds and optically pumped operation limit their applications in silicon photonics. In this regards, the concept of a highperformance III-V laser monolithically integrated on Si substrate has attracted enormous research interests as a promising approach for an on-chip light source in Si photonics, because III-V compounds are one of the ideal gain materials to generate coherent light owing to their superior optical properties.

For growing high quality III-V material on $\mathrm{Si}$, a great deal of efforts has been made to tackle the challenges such as high density of threading dislocations, antiphase domains (APD) and thermal cracks. As a result, high-performance III-V lasers with optimal buffer layers can be successfully grown on lattice mismatched Si substrate [1, 3]. In this paper, we first describe high-performance and high-reliability 1300nm InAs/GaAs QD laser monolithically grown on Si substrates, and then, recent progress on Si-based QD III-V laser with different structures is presented.

\section{QD lasers monolithically integrated on $\mathrm{Si}$ substrate}

2.1. Defect tolerance in InAs/GaAs quantum dot lasers grown on silicon

QD lasers grown on Si substrate not only benefit from the advantages of low $\mathrm{J}_{\text {th }}$ and high temperature performance, but also provide the property of insensitivity to dislocations and defects. In order to prove that the QD is a promising material for Si-based light source over QW, we present a study on the impact of threading dislocations on III-V QD and QW lasers monolithically grown on Si substrates under the same conditions [1]. As shown in Fig.1 (a), the QD laser shows high performance with an output power of over $100 \mathrm{~mW}$ and a threshold current density $\left(\mathrm{J}_{\mathrm{th}}\right)$ of $160 \mathrm{~A} / \mathrm{cm}^{2}$ under continuous-wave $(\mathrm{CW})$ operation and room temperature $(\mathrm{RT})$. In contrast, there is no lasing behavior for the QW laser devices.
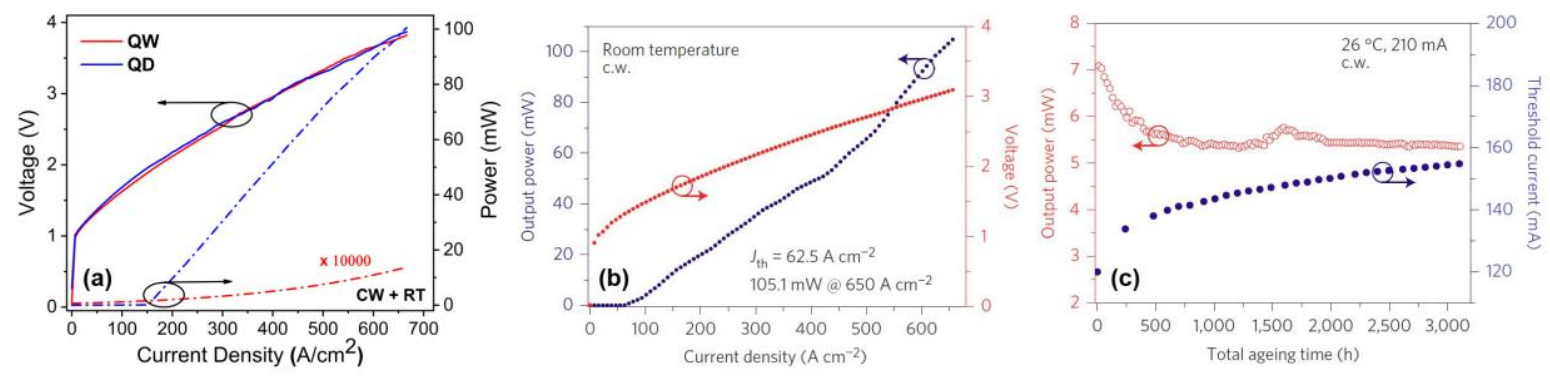

Fig.1 (a) LIV results of $Q D$ and $Q W$ lasers monolithically grown on Si substrate under the same growth conditions. (b) LIV curve of InAs/GaAs $Q D$ lasers on Si substrate under CW operation and room temperature. (c) Aging test result of Si-based QD laser. 


\subsection{Long life III-V QD lasers grown on Si substrates}

An InAs/GaAs QD laser directly grown on Si substrate with long lifetime and low threshold current density was demonstrated in 2016 [4]. In this report, $4^{\circ}$ offcut $\mathrm{Si}$ substrate towards the [011] plane was used to eliminate the APDs. Besides, both an AlAs nucleation layer and optimized InGaAs/GaAs dislocation filter layers (DFLs) are adopted to further reduce the dislocation density. As shown in Fig.1 (b), the threshold current density of this QD laser is only $62.5 \mathrm{~A} / \mathrm{cm}^{2}$ which is comparable with that of GaAs-based QD lasers. In addition, an aging test over 3100 hours at a constant heatsink temperature of $26^{\circ} \mathrm{C}$ and driving current of $210 \mathrm{~mA}$ is conducted, as shown in Fig.1 (c). The calculated lifetime under sublinear model is estimated to be over 100,158 hours, indicating outstanding application value of QD lasers on $\mathrm{Si}$.

\section{Recent progress on Si-based InAs/GaAs QD laser}

\subsection{Low noise $1300 \mathrm{~nm}$ InAs/GaAs QD laser grown on silicon with narrow ridge waveguide structure}

To realize single transverse mode and low threshold current of Si-based light source, $2.2 \mu$ midth narrow ridge waveguide InAs/GaAs QD laser devices with 95\% high reflection coating were fabricated [5]. Fig.2 (a) shows the LIV measurement of a $2.2 \mu \mathrm{m} \times 2.5 \mathrm{~mm} \mathrm{Si-based} \mathrm{narrow-ridge-waveguide} \mathrm{laser} \mathrm{device.} \mathrm{The} \mathrm{output} \mathrm{power} \mathrm{of} 25$ $\mathrm{mW}$ and the threshold current as low as $12.5 \mathrm{~mA}$ under $\mathrm{CW}$ and $\mathrm{RT}$ were achieved.

Normally, the Si electronic chips employed in Si-based photonic electronic integration require high temperature operation at $65^{\circ} \mathrm{C}$ or above $[4,5]$. For practical applications, accordingly, $\mathrm{CW}$ lasing operation at high temperature is indispensable. Fig.2 (b) demonstrates the temperature dependent L-I curve of a $2.2 \mu \mathrm{m} \times 4$ mm narrow-ridgewaveguide laser under $\mathrm{CW}$ operation. It is shown that the device is able to operate up to $90^{\circ} \mathrm{C}$.

Based on $2.2 \mu \mathrm{m}$ width narrow ridge laser devices, the ultra-low relative intensity noise (RIN) was performed. As shown in Fig. 2 (c), the RIN was measured to be lower than $-150 \mathrm{dBm} / \mathrm{Hz}$ when the bias current is more than 60 $\mathrm{mA}$. This result indicates that low noise $\mathrm{Si}$-based lasers can be a promising light source for data interconnections.
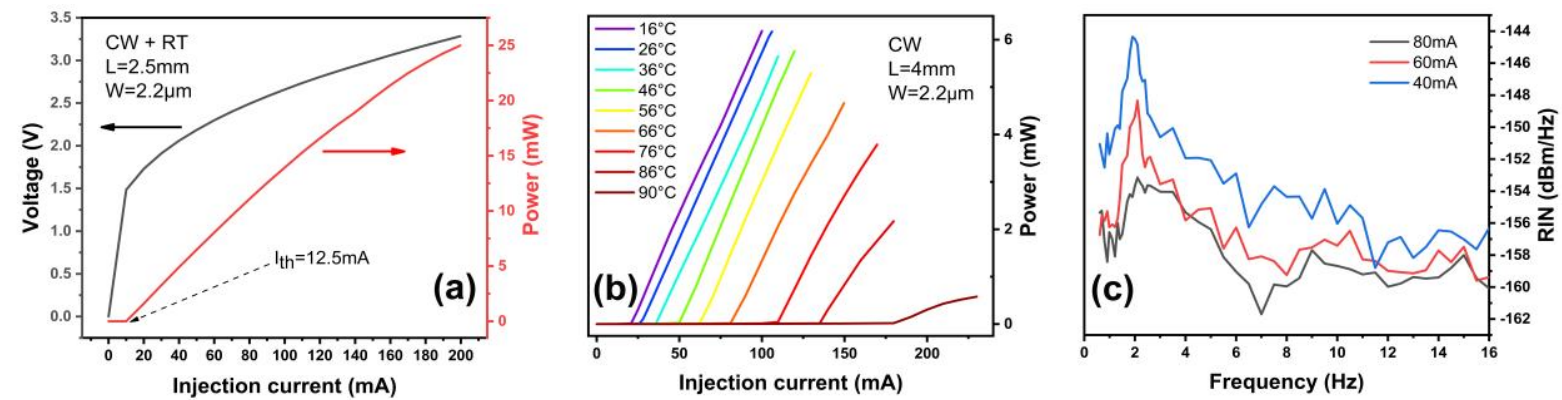

Fig.2 (a) LIV results of a $2.2 \mu \mathrm{m} \times 2.5 \mathrm{~mm}$ narrow-ridge-waveguide QD lasers monolithically grown on Si substrate under RT and CW. (b) Temperature dependence measured L-I curve from a $2.2 \mu \mathrm{m} \times 4 \mathrm{~mm}$ narrow-ridge-waveguide laser under CW mode. (c) The RIN spectrum measured from $0.5 \mathrm{GHz}$ to $16 \mathrm{GHz}$ at gain currents of 40, 60, and $80 \mathrm{~mA}$.

\subsection{Monolithic $Q D$ distributed feedback $(D F B)$ laser array on silicon}

Single mode lasers are required for advanced communication system to realize wavelength division multiplexing, and distributed feedback laser array is known as an effective way to obtain a single mode light source. Recently, the first electrically pumped InAs/GaAs QD DFB laser array epitaxially grown on silicon was presented [6].
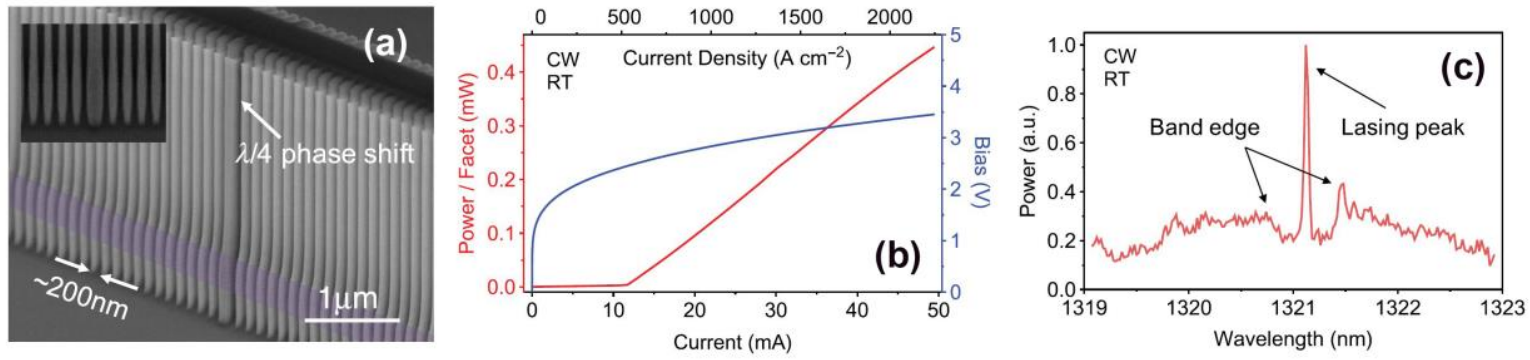

Fig.3 (a) SEM image of the grating structure and the N4 phase shift. (b) LIV curve of InAs/GaAs QD DFB lasers on Si substrate under CW operation and RT. (c) Spectrum of the DFB laser operating around threshold. Resolution: $0.07 \mathrm{~nm}$. 
In this research, the $\lambda / 4$ phase shift was used to force single-longitudinal-mode lasing in the defect mode. Fig.3 (a) presents the SEM image of the grating with the $\lambda / 4$ phase shift. As can be seen in Fig.3 (b), the threshold current of the DFB laser is $12.5 \mathrm{~mA}$ and the calculated threshold current density is around $550 \mathrm{~A} / \mathrm{cm}^{2}$ under RT and CW mode. While keeping the injection current around threshold current, the spectrum of the DFB device is plotted in Fig.3 (c), which matches the typical $\lambda / 4$ phase-shifted DFB laser characteristics. The Si-based DFB laser devices are able to meet the requirement of single mode light source for silicon photonics, and this research is also useful for non-communications applications such as on-chip sensing and metrology.

\subsection{Ultra-low threshold InAs/GaAs QD microdisk lasers on on-axis Si (001)}

Compared with ridge-waveguide laser devices, the small size of micro-lasers leads to great potential to gain lower power consumption and high reliability [7]. Whispering-gallery-mode microdisk lasers are an ideal light source for silicon photonics due to the small footprint configurations and ultra-low threshold. Last year, an InAs/GaAs QD microdisk lasers grown on on-axis $\mathrm{Si}$ (001) substrate with ultra-low threshold was reported [7].
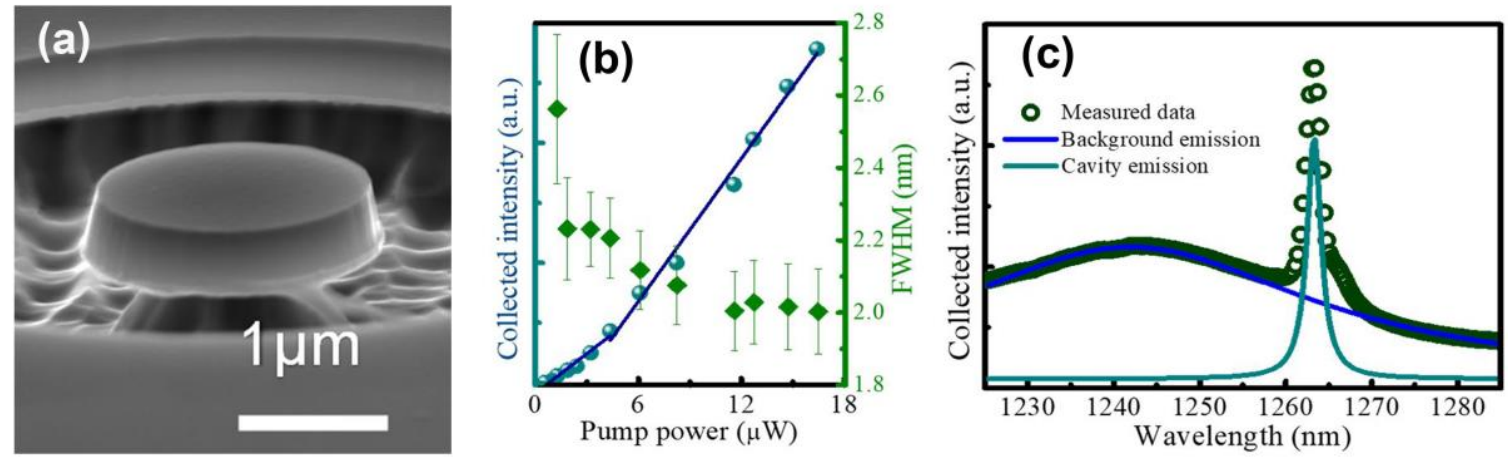

Fig.4 (a) SEM image of the microdisk laser after fabrication. (b) Corresponding collected intensity and mode linewidth as a function of input power for the emission peak at $1263 \mathrm{~nm}$. (c) Spectrum above the threshold $(4.4 \mu \mathrm{W})$ and curve fitting showing the spontaneous emission background and cavity emission.

The SEM image of the fabricated microdisk laser with diameter of $1.9 \mu \mathrm{m}$ is shown in Fig.4 (a). It is obvious that the surface of the active region is smooth enough. As shown in Fig.4 (b), the lasing threshold of the Si-based microdisk laser is around $2.6 \mathrm{~mW}$ under $\mathrm{CW}$ optical pump, which is an ultra-low value among all publications. From the spectrum result shown in Fig.4 (c), the spectrum curve is separated into background emission and cavity emission fitting curves.

The impressive lasing characteristics of the microdisk lasers monolithically grown on $\mathrm{Si}(001)$ substrate with an ultra-low threshold and small footprint are a major progress in Si-based light sources towards the low-cost and large-scale integration of silicon photonics.

\section{References}

[1] Z Liu, C Hantschmann, M Tang, Y Lu, J Park, M Liao, S Pan, A Sanchez, R Beanland, M Martin, T Baron, S Chen, A Seeds, R Penty, I White, and H Liu, "Origin of defect tolerance in InAs / GaAs quantum dot lasers grown on silicon," IEEE J. Light. Technol., DOI: 10.1109/JLT.2019.2925598, vol. PP, p. 1, 2019.

[2] M. Liao, S. Chen, S. Huo, S. Chen, J. Wu, M. Tang, K. Kennedy, W. Li, S. Kumar, M. Martin, T. Baron, C. Jin, I. Ross, A. Seeds, and H. Liu, "Monolithically integrated electrically pumped continuous-wave III-V quantum dot light sources on silicon," IEEE J. Sel. Top. Quantum Electron. $23,1-10,2017$.

[3] S. Chen, M. Liao, M. Tang, J. Wu, M. Martin, T. Baron, A. Seeds, and H. Liu, "InAs / GaAs quantum dot lasers monolithically grown on onaxis Si (001) substrates," Optics Express, vol. 25, no. 5, pp. 11381-11386, 2017.

[4] S. Chen, W. Li, J. Wu, Q. Jiang, M. Tang, S. Shutts, S. Elliott, A. Sobiesierski, A. Seeds, I. Ross, P. Smowton, and H. Liu, "Electrically pumped continuous-wave III-V quantum dot lasers on silicon," Nat. Photonics, vol. 10, no. 5, pp. 307-311, 2016.

[5] M Liao, S Chen, Z Liu, Y Wang, L Ponnsmpslsm, Z Zhou, J Wu, M Tang, S Shutts, Z Liu, P Smowton, S Yu, A Seeds, and H Liu, "Lownoise $1.3 \mu \mathrm{m} \mathrm{InAs}$ / GaAs quantum dot laser monolithically grown on silicon," Photonics Research, vol. 6, no. 11, pp. 1062-1066, 2018.

[6] Y Wang, S Chen, Y Yu, L Zhou, L Liu, C Yang, M Liao, M Tang, Z Liu, J Wu, W Li, I Ross, A Seeds, H Liu, and S Yu, "Monolithic quantum-dot distributed feedback laser array on silicon," Optica, vol. 5, no. 5, pp. 528-533, 2018.

[7] T Zhou, M Tang, G Xiang, X Fang, X Liu, B Xiang, S Hark, M Martin, M Touraton, T Baron, Y Lu, S Chen, H Liu, and Z Zhang, "Ultra-low threshold InAs / GaAs quantum dot microdisk lasers on planar on-axis Si (001) substrates," Optica, vol. 6, no. 4, 2019. 